ARTICLE

DOI: $10.1038 / s 41467-018-05482-0$

\title{
Nucleoside-modified mRNA immunization elicits influenza virus hemagglutinin stalk-specific antibodies
}

Norbert Pardi ${ }^{1}$, Kaela Parkhouse ${ }^{2}$, Ericka Kirkpatrick ${ }^{3,4}$, Meagan McMahon $^{3}$, Seth J. Zost ${ }^{2}$, Barbara L. Mui ${ }^{5}$, Ying K. Tam ${ }^{5}$, Katalin Karikó ${ }^{6}$, Christopher J. Barbosa ${ }^{5}$, Thomas D. Madden ${ }^{5}$, Michael J. Hope ${ }^{5}$, Florian Krammer ${ }^{3}$, Scott E. Hensley ${ }^{2} \&$ Drew Weissman ${ }^{1}$

Currently available influenza virus vaccines have inadequate effectiveness and are reformulated annually due to viral antigenic drift. Thus, development of a vaccine that confers long-term protective immunity against antigenically distant influenza virus strains is urgently needed. The highly conserved influenza virus hemagglutinin (HA) stalk represents one of the potential targets of broadly protective/universal influenza virus vaccines. Here, we evaluate a potent broadly protective influenza virus vaccine candidate that uses nucleoside-modified and purified mRNA encoding full-length influenza virus HA formulated in lipid nanoparticles (LNPs). We demonstrate that immunization with HA mRNA-LNPs induces antibody responses against the HA stalk domain of influenza virus in mice, rabbits, and ferrets. The HA stalk-specific antibody response is associated with protection from homologous, heterologous, and heterosubtypic influenza virus infection in mice.

\footnotetext{
${ }^{1}$ Department of Medicine, University of Pennsylvania, Philadelphia, PA 19104, USA. ${ }^{2}$ Department of Microbiology, Perelman School of Medicine, University of Pennsylvania, Philadelphia, PA 19104, USA. ${ }^{3}$ Department of Microbiology, Icahn School of Medicine at Mount Sinai, New York, NY 10029, USA. ${ }^{4}$ Graduate School of Biomedical Sciences, Icahn School of Medicine at Mount Sinai, New York, NY 10029, USA. ${ }^{5}$ Acuitas Therapeutics, Vancouver, BC V6T 1Z3, Canada. ${ }^{6}$ BioNTech RNA Pharmaceuticals, An der Goldgrube 12, 55131 Mainz, Germany. These authors contributed equally: Norbert Pardi, Kaela Parkhouse. These authors jointly supervised this work: Scott E. Hensley, Drew Weissman. Correspondence and requests for materials should be addressed to S.E.H. (email: hensley@pennmedicine.upenn.edu) or to D.W. (email: dreww@pennmedicine.upenn.edu)
} 
$\mathrm{S}$ easonal influenza virus epidemics pose a significant global health threat. Inactivated and live attenuated vaccines have limited effectiveness and need to be reformulated every year. These vaccines induce antibody responses primarily against the immunodominant globular head domain of influenza virus hemagglutinin (HA), but the virus can easily escape from protective immune responses due to the plasticity of the HA head (reviewed in ref. ${ }^{1}$ ). It may be possible to induce broader protection with vaccines that target more conserved viral regions, such as the stalk domain of HA that is less tolerant of escape mutations (reviewed in ref. ${ }^{2}$ ). In recent years, several HA immunogens have been developed that elicit HA stalk-specific immune responses. For example, headless HAs and chimeric HAs (cHAs) induce potent stalk-reactive antibodies ${ }^{3-7}$. Heterologous prime-boost immunizations were also shown to elicit HA stalkspecific antibodies in preclinical studies ${ }^{6-11}$. Notably, most HA stalk-based vaccines require multiple immunizations.

In vitro-transcribed messenger RNA (mRNA)-based vaccines have shown promise against cancer and infectious diseases (reviewed in ref. ${ }^{12}$ ). For example, a lipid nanoparticle (LNP) encapsulated $^{13} 1$-methylpseudouridine-modified mRNA vaccine protected mice and non-human primates against Zika virus infection after a single low dose immunization ${ }^{14}$. Although, several recent studies indicate that mRNA-based vaccines can provide protection against influenza virus infection, none of these reports determined if mRNA-based influenza virus vaccines elicited broadly reactive antibodies capable of neutralizing antigenically distinct influenza virus strains after a single immunization ${ }^{15-19}$

Here, we demonstrate that vaccination with influenza virus HA-encoding, nucleoside-modified ${ }^{20}$, and fast protein liquid chromatography (FPLC)-purified ${ }^{21}$ mRNA-LNPs induces potent antibody responses that target the conserved HA stalk domain in mice, rabbits, and ferrets. These broadly reactive antibody responses were associated with protection from homologous, heterologous, and heterosubtypic influenza viruses in mice. We propose that nucleoside-modified, FPLC-purified mRNA-LNP vaccines represent a promising broadly protective influenza virus vaccine candidate.

\section{Results}

HA head and stalk-specific antibody responses in mice. To evaluate the immunogenicity of the nucleoside-modified HA mRNA-LNP vaccine, mice were immunized twice with 3,10 , or $30 \mu \mathrm{g}$ of A/California/07/2009 (H1N1) (A/Cal09) HA-encoding mRNA-LNPs intradermally (i.d.) or 10,30 , or $90 \mu \mathrm{g}$ of A/California/07/2009 HA mRNA-LNPs intramuscularly (i.m.) and antibody responses were assessed. The two immunizations were delivered 4 weeks apart. Control animals were vaccinated with $30 \mu \mathrm{g}$ (i.d.) or $90 \mu \mathrm{g}$ (i.m.) of poly(C) RNA-LNPs. A single immunization induced potent antibody responses targeting the HA globular head domain as determined by hemagglutination inhibition (HAI) assays using the homologous A/California/07/2009 virus (Fig. 1a, b). Higher vaccine doses elicited higher HAI titers with subtle differences between the i.d. and i.m.-immunized animals. A second immunization substantially increased HAI titers that reached 1:1280-1:20,480 at week 8 (Fig. 1a, b). As expected, antibodies elicited by the A/California/07/2009 HA mRNA-LNPs had no HAI activity against the A/Puerto Rico/8/1934 H1N1 virus (Fig. 1c, d), which possesses a genetically divergent HA globular head domain (Supplementary Fig. 1).

We next performed enzyme-linked immunosorbent assays (ELISAs) using cHA antigens to quantify $\mathrm{HA}$ stalk-reactive
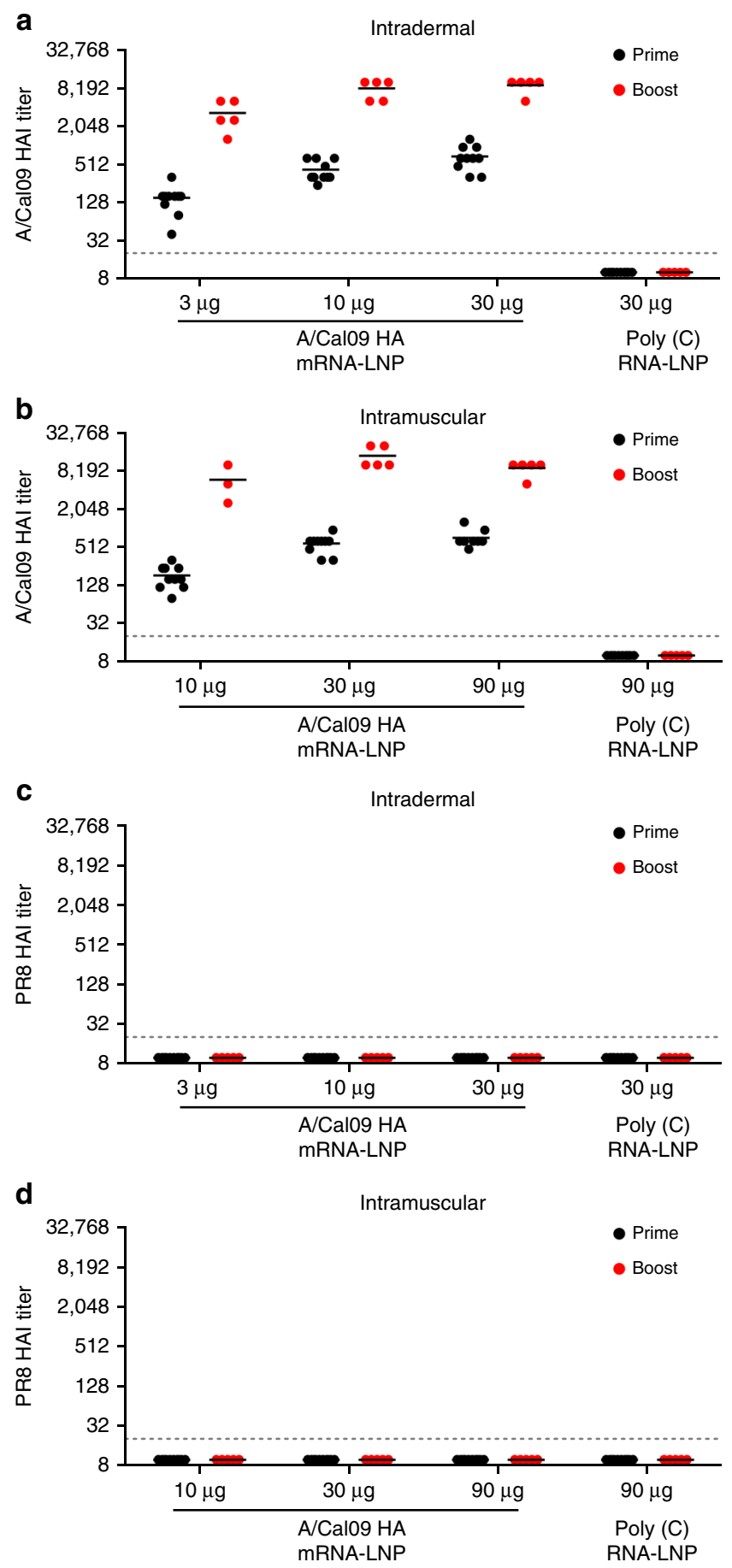

Fig. 1 Nucleoside-modified HA mRNA-LNP immunization elicits potent neutralizing antibody responses in mice. Mice received two i.d. $(3,10$, or $30 \mu \mathrm{g})$ or i.m. (10,30, or $90 \mu \mathrm{g})$ immunizations of A/California/07/2009 HA mRNA-LNPs or 30 (i.d.) or 90 (i.m.) $\mu$ g of poly (C) RNA-LNPs at week 0 (prime) and 4 (boost). HA inhibition (HAI) titers against A/California/07/ 2009 (a, b) and A/Puerto Rico/8/1934 (c, d) viruses were determined at week 4 and $8 . n=3-10$ mice and each symbol represents one animal. Two independent experiments were performed. Horizontal lines show the mean; dotted line indicates the limit of detection. Statistical analysis: $\mathbf{a}, \mathbf{b}$ two-way ANOVA with Bonferroni correction on log-transformed data, $p<0.05$; all comparisons across doses and time points were statistically significant except for (a) $10 \mu \mathrm{g}$ HA prime vs. $30 \mu \mathrm{g} \mathrm{HA}$ prime and $10 \mu \mathrm{g}$ HA boost vs. $30 \mu \mathrm{g} \mathrm{HA}$ boost and for (b) $10 \mu \mathrm{g}$ HA boost vs. $90 \mu \mathrm{g} \mathrm{HA}$ boost, $30 \mu \mathrm{g} \mathrm{HA}$ prime vs. $90 \mu \mathrm{g} \mathrm{HA}$ prime and $30 \mu \mathrm{g}$ HA boost vs. $90 \mu \mathrm{g} \mathrm{HA}$ boost 

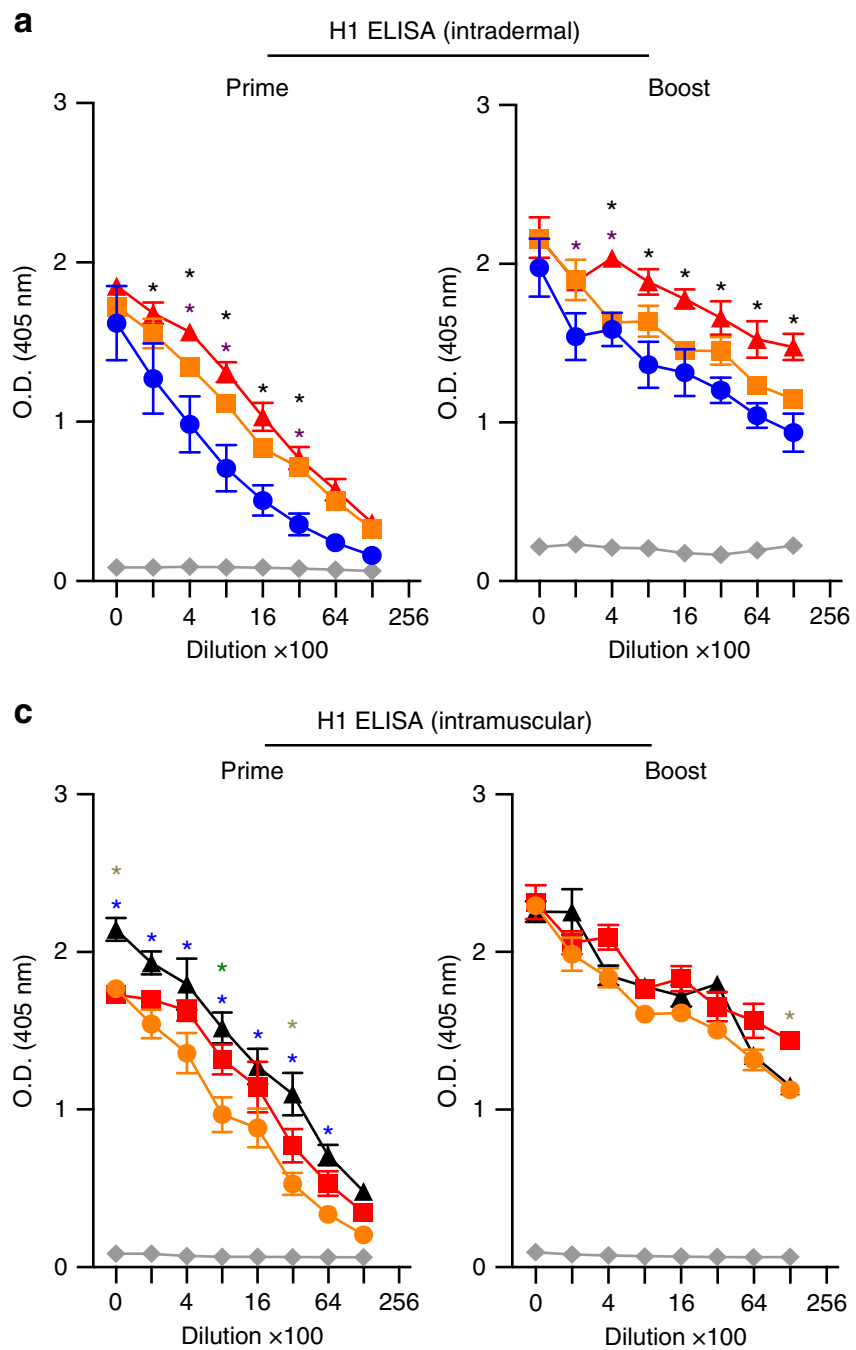

Fig. 2 Nucleoside-modified HA mRNA-LNP immunization elicits HA stalk-specific antibody responses in mice. Mice received two i.d. (3, 10, or 30 $\mu$ g) (a, b) or i.m. $(10,30$, or $90 \mu \mathrm{g})(\mathbf{c}, \mathbf{d})$ immunizations of A/California/07/2009 HA mRNA-LNPs or 30 (i.d.) or 90 (i.m.) $\mu \mathrm{g}$ of poly(C) RNA-LNPs at weeks 0 and 4. IgG binding to full-length $\mathrm{H} 1 \mathrm{HA}(\mathbf{a}, \mathbf{c})$ and $\mathrm{cH} 6 / 1 \mathrm{HA}(\mathbf{b}, \mathbf{d})$ proteins was determined at week 4 and week $8 . n=3-10$ mice. Two independent experiments were performed. Error bars are SEM. Statistical analysis: $\mathbf{a}, \mathbf{b}$ two-way ANOVA with Bonferroni correction, ${ }^{*} p<0.05$ comparing A/California/ 07/2009 HA mRNA-LNP dose groups at each serum dilution, black star: significant difference in titers between the 3 and $30 \mu \mathrm{g}$ groups, purple star: significant difference in titers between the 3 and $10 \mu$ groups, green star: significant difference in titers between the 10 and $30 \mu g$ groups; c, d two-way ANOVA with Bonferroni correction, ${ }^{\star} p<0.05$ comparing A/California/07/2009 HA mRNA-LNP dose groups at each serum dilution, blue star: significant difference in titers between the 10 and $90 \mu \mathrm{g}$ groups, green star: significant difference in titers between the 10 and $30 \mu g$ groups, gray star: significant difference in titers between the 30 and $90 \mu$ groups

antibodies elicited by nucleoside-modified HA mRNA-LNP vaccination. For these experiments, we measured antibody binding to full-length $\mathrm{A} /$ California/07/2009 $\mathrm{HA}$ and an $\mathrm{H} 6 / 1$ cHA (cH6/1 HA) that possesses an H1 stalk domain and an H6 "exotic" globular head domain. Previous studies have shown that $\mathrm{H} 1$ stalk-reactive antibodies bind to both the full-length $\mathrm{H} 1$ construct and the cH6/1 construct, whereas H1 globular headreactive antibodies bind only to the full-length $\mathrm{H} 1$ construct and not the $\mathrm{cH} 6 / 1$ construct $^{22}$. Antibodies from all A/California/07/ 2009 HA-vaccinated mice showed very strong binding to fulllength A/California/07/2009 HA after a single immunization (Fig. 2a, c). Importantly, HA stalk-reactive antibodies capable of binding to $\mathrm{cH} 6 / 1 \mathrm{HA}$ were also elicited by a single immunization. A booster immunization significantly increased HA stalk-reactive antibodies capable of binding to $\mathrm{cH} 6 / 1 \mathrm{HA}$ (Fig. 2b, d).

To determine if HA stalk-specific antibodies could be elicited with a different influenza HA immunogen, mice were i.d.

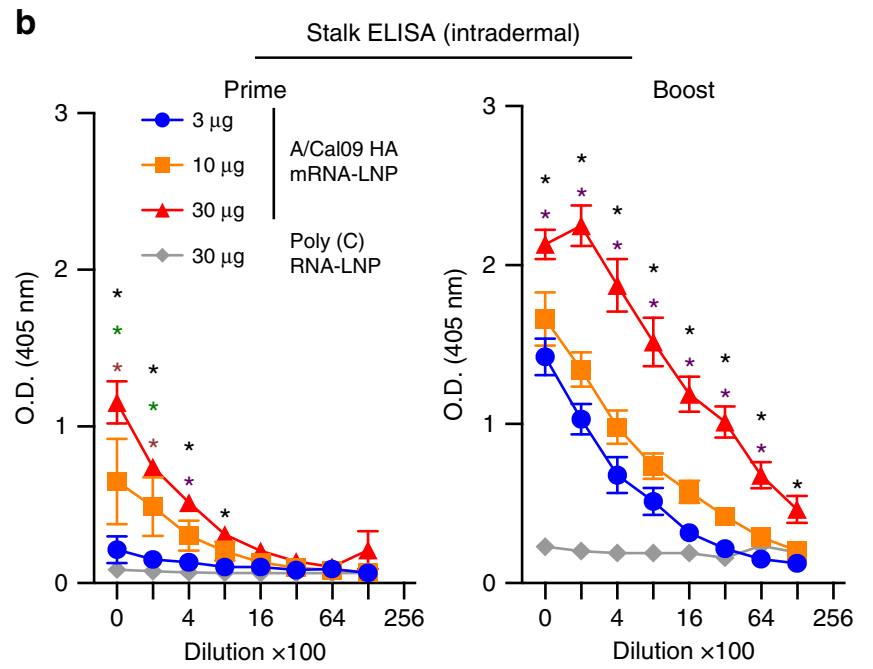

d

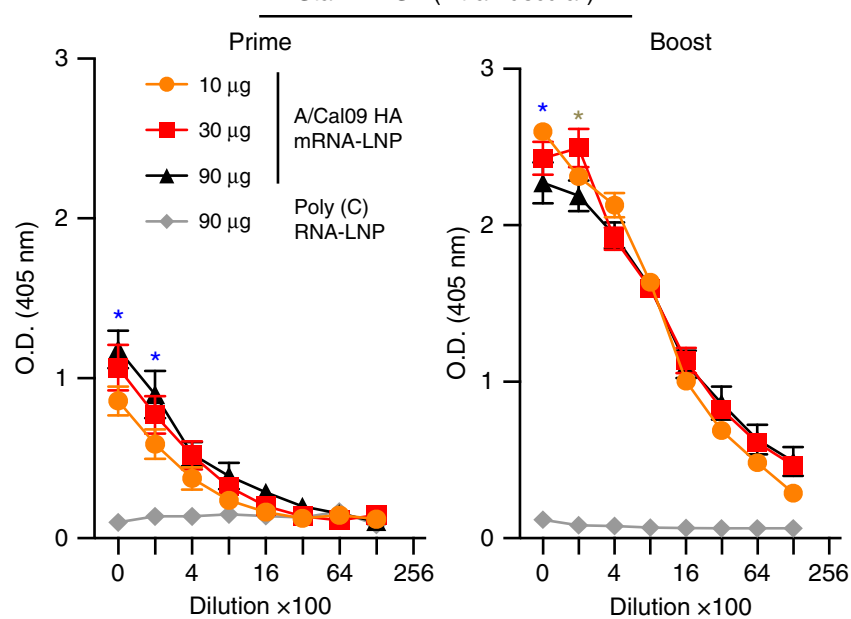


a

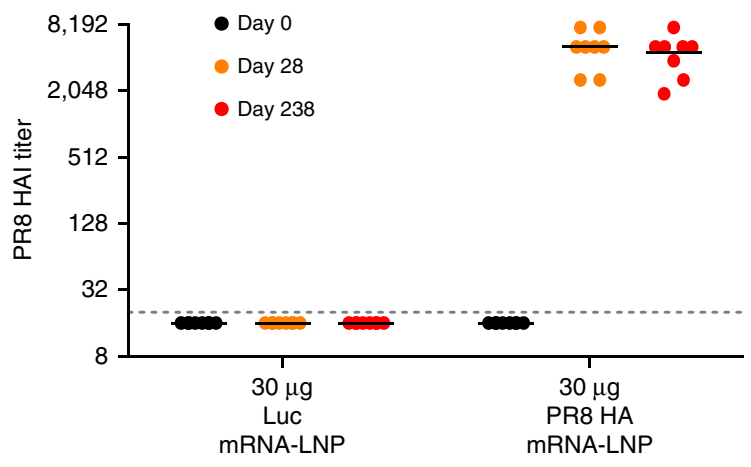

b

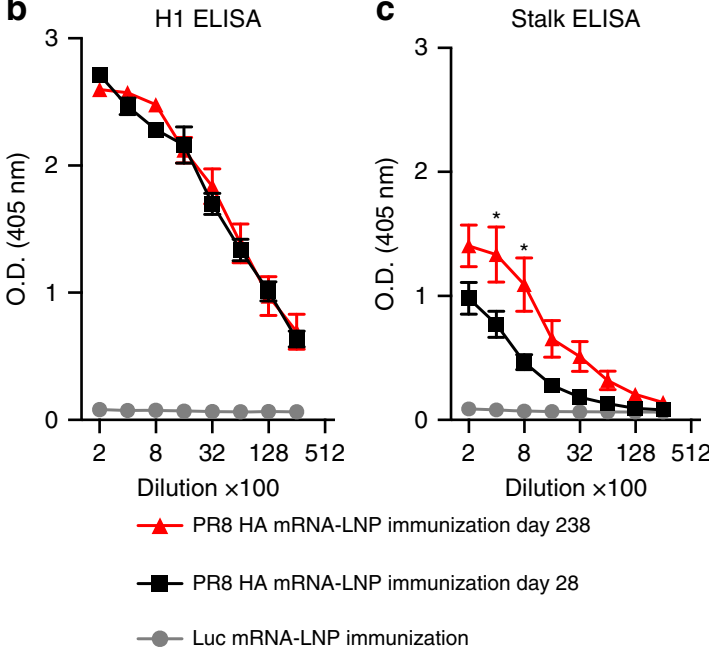

Fig. 3 Nucleoside-modified HA mRNA-LNP immunization elicits durable HA stalk-specific antibody responses in mice. Mice received a single i.d. dose of $30 \mu \mathrm{g}$ of A/Puerto Rico/8/1934 HA mRNA-LNP vaccine and a HAl titers against A/Puerto Rico/8/1934 and lgG binding to full-length H1 HA (b) and cH5/ $1 \mathrm{HA}(\mathbf{c})$ proteins in mouse serum obtained 28 and 238 days post single immunization were determined. $n=8$ mice. a Each symbol represents one animal, horizontal lines show the mean, dotted line indicates the limit of detection. b, c Error bars are SEM. Statistical analysis: a two-way ANOVA with Bonferroni correction on log-transformed data, $p<0.05$; all comparisons between the Luc and the day 28 and day 238 Puerto Rico/8/1934 HA groups were statistically significant. b, c Two-way ANOVA with Bonferroni correction comparing Puerto Rico/8/1934 HA day 28 and day 238 time points for different dilutions. ${ }^{\star} p<0.05$

nucleoside-modified mRNA-LNP vaccination. Mice were immunized with a single $30 \mu \mathrm{g}$ dose of A/California/07/2009 HAencoding mRNA-LNPs i.d. and $\mathrm{CD}^{+} \mathrm{T}$ cell responses were determined 12 days later. As a comparator, a group of mice were immunized with $3 \mu \mathrm{g}$ of monovalent A/California/07/2009 virus vaccine. Unlike inactivated virus immunization, HA mRNA-LNP vaccination induced $\mathrm{HA}$-specific $\mathrm{CD}^{+}{ }^{+} \mathrm{T}$ cell responses as measured by intracellular cytokine production (Supplementary Fig. 2).

HA stalk-reactive antibody responses in rabbits and ferrets. To further evaluate the potency of the nucleoside-modified mRNALNP influenza virus vaccine, immunogenicity was also evaluated in rabbits and ferrets. Rabbits were i.d. immunized twice with $50 \mu \mathrm{g}$ of A/California/07/2009 HA mRNA-LNPs and antibody responses were evaluated. A single immunization elicited HAI titers ranging from 1:120 to 1:320, as well as, HA stalk-specific antibody responses (Supplementary Fig. 3). A second immunization significantly boosted antibody responses and resulted in increased HAI titers and stalk-specific antibodies (Supplementary Fig. 3).

Next, we evaluated the nucleoside-modified mRNA-LNP influenza virus vaccine in a ferret model. Animals were immunized i.m. twice with $60 \mu \mathrm{g}$ of A/California/07/2009 HA mRNA-LNPs. Antibodies against the HA globular head domain were elicited in 8 out of 12 ferrets ( $\geq 1: 40 \mathrm{HAI}$ in all responders) 4 weeks after the first immunization as measured by HAI assays (Fig. 4a). Substantially increased HAI titers $(\geq 1: 160$ in 10 out of 12 animals) were obtained after the boost. No HAI activity against the heterologous asH1N1 (A/swine/Jiangsu/40/2011, Supplementary Fig. 4) virus was detected (Fig. 4b). Importantly, animals generated HA stalk-reactive antibodies that bound to the cH6/1 HA protein (Fig. 4c). Moreover, as measured by in vitro microneutralization ( $\mathrm{MN})$ experiments, sera obtained from ferrets 4 weeks after the first and 9 weeks after the second immunization neutralized the closely related pH1N1 (A/Netherlands/602/2009) and the more antigenically distant asH1N1 virus (Supplementary Fig. 5).
Collectively, these studies indicate that nucleoside-modified HA mRNA-LNP vaccines elicit high levels of antibodies against both the HA head and stalk domains of influenza virus in mice, rabbits, and ferrets.

Protection from homologous and heterologous viruses in mice. To investigate the protective efficacy of mRNA immunization, mice that were immunized with two i.d. or i.m. doses of A/ California/07/2009 HA mRNA-LNPs were challenged with the homologous A/California/07/2009 H1N1 virus or the heterologous A/Puerto Rico/8/1934 H1N1 virus 5 weeks after the last immunization. Both challenge viruses possessed A/Puerto Rico/8/ 1934 internal genes and the A/California/7/2009 HA possessed a D225G mutation to facilitate viral replication in mice. All HA mRNA-LNP-vaccinated animals were protected from both the homologous (Fig. 5a, b) and heterologous $\mathrm{H} 1 \mathrm{~N} 1$ virus infection (Fig. 5c, d), although some initial weight loss in the low dose groups was observed after challenge with the A/Puerto Rico/8/ 1934 virus (Fig. 5c). Mice injected with control poly(C) RNALNP lost weight and died or needed to be euthanized after viral challenge (Fig. 5).

Based on the high HAI titers and measurable HA stalk-reactive antibody responses elicited by the mRNA-LNP constructs, we hypothesized that a single HA mRNA-LNP immunization could elicit protective immunity. Mice were i.m. immunized with a single dose of $30 \mu \mathrm{g}$ of A/California/07/2009 HA mRNA-LNPs or $30 \mu \mathrm{g}$ of poly $(\mathrm{C}) \mathrm{RNA}-\mathrm{LNPs}$. As a comparator, a group of mice were immunized with $3 \mu \mathrm{g}$ of monovalent A/California/07/2009 virus vaccine. Four weeks after vaccination, mRNA-LNP vaccines induced HAI titers ranging between 320-960 against the autologous strain, whereas the monovalent virus vaccine elicited very low HAI titers of $\sim 1: 15$ (Fig. 6a). No HAI activity against the PR8 virus was detected (Fig. 6a). High antibody titers elicited by the mRNA-LNP vaccine were associated with protection following challenge with the homologous A/California/07/2009 viral strain and the heterologous A/Puerto Rico/8/1934 viral strain (Fig. 6b, c). All mRNA-LNP-immunized and monovalent virus vaccine-injected animals survived A/ 


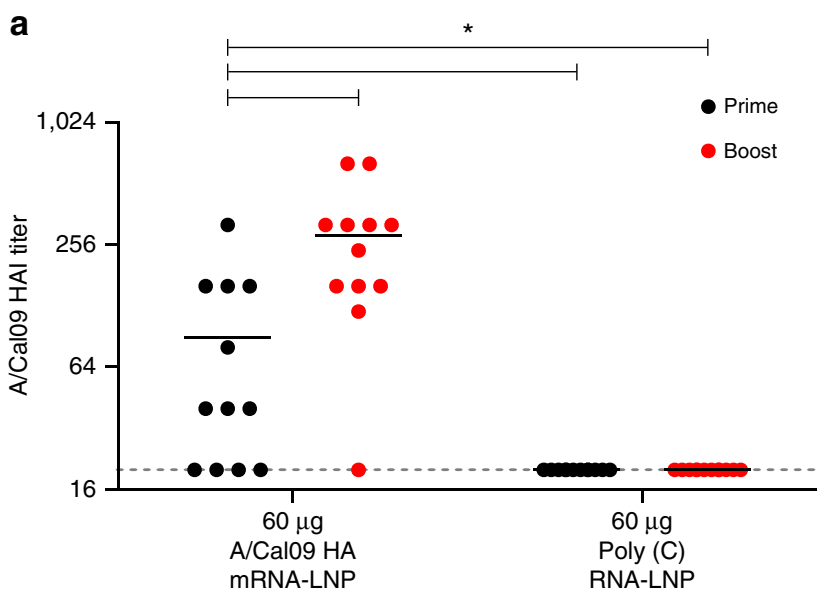

b
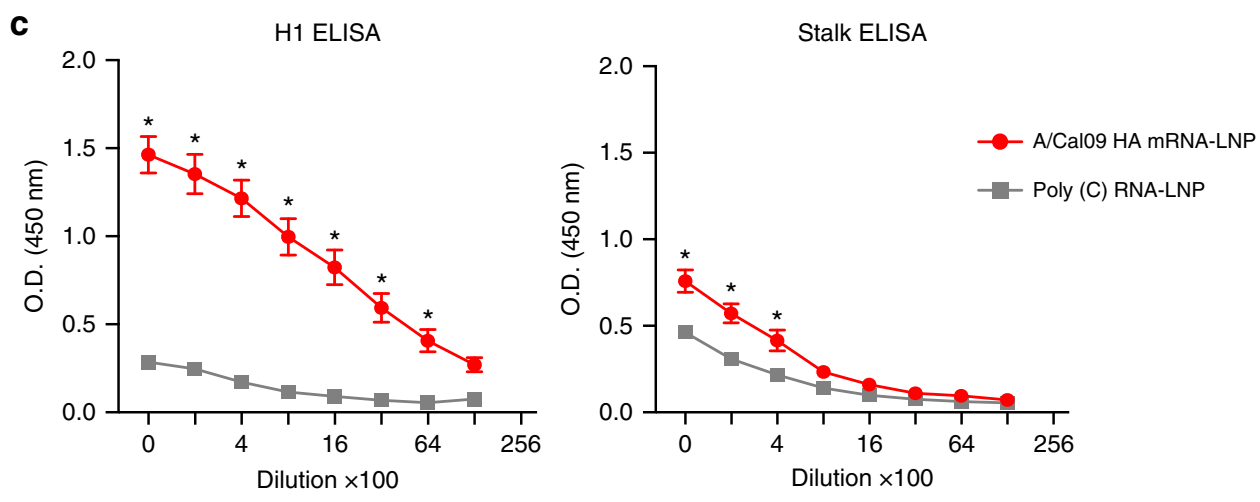

Fig. 4 Nucleoside-modified HA mRNA-LNP immunization induces HA stalk-reactive antibodies in ferrets. Ferrets were immunized two times i.m. with $60 \mu \mathrm{g}$ of A/California/07/2009 HA mRNA-LNPs or $60 \mu \mathrm{g}$ of poly(C) RNA-LNPs at week 0 (prime) and 4 (boost). HAl titers against the A/California/07/ 2009 (a) and A/swine/Jiangsu/40/2011 (b) viruses were determined at week 4 (prime) and week 13 (boost). c lgG binding to full-length $\mathrm{H} 1 \mathrm{HA}$ (total) and $\mathrm{cH} 6 / 1 \mathrm{HA}$ (stalk) proteins from serum samples obtained 9 weeks after the second immunization was determined. $n=12$ ferrets. $\mathbf{a}, \mathbf{b}$ Each symbol represents one animal, horizontal lines show the mean, dotted line indicates the limit of detection. c error bars are SEM. Statistical analysis: $\mathbf{a}, \mathbf{b}$ one-way ANOVA with Bonferroni correction on log-transformed data, ${ }^{\star} p<0.05$. c Two-way ANOVA with Bonferroni correction comparing A/California/07/2009 $\mathrm{HA}$ and poly $(\mathrm{C})$ immunizations for different dilutions. ${ }^{*} p<0.05$

California/07/2009 virus infection; however, animals in both groups lost a substantial amount of weight following infection (Fig. 6b). mRNA-LNP-immunized animals displayed weight loss following A/Puerto Rico/8/1934 virus challenge, but they rapidly recovered and survived virus infection (Fig. 6c). In contrast, mice that were immunized with the monovalent A/California/07/2009 virus vaccine or the poly(C) RNA-LNP vaccine rapidly developed symptoms and $100 \%$ of the animals died following A/Puerto Rico/8/1934 virus challenge (Fig. 6c). These data demonstrate that a single mRNA-LNP immunization induced protection from an antigenically distant $\mathrm{H} 1$ virus (Supplementary Fig. 1) in the absence of HAI activity to this viral HA.

Protection from a heterosubtypic virus in mice. We next performed a series of studies with an $\mathrm{H} 5 \mathrm{~N} 1$ influenza virus to determine whether nucleoside-modified HA mRNA-LNP immunization elicits protective immune responses against more antigenically distant influenza virus subtypes. Mice were immunized twice with $30 \mu \mathrm{g}$ of A/California/07/2009 HA mRNA-LNPs i.d. or $1 \mu \mathrm{g}$ (total protein) of inactivated $\mathrm{H} 5 \mathrm{~N} 1$ influenza virus i. m. ${ }^{24}$. Mice were then challenged with a lethal dose of $\mathrm{H} 5 \mathrm{~N} 1$ virus (6:2 reassortant between A/Puerto Rico/8/1934 and A/Vietnam/ $1203 / 04$, which donated HA and neuraminidase) 4 weeks after the second immunization and weight and morbidity were monitored for 14 days. Strikingly, all HA mRNA-LNP-immunized animals survived viral challenge, while those injected with poly (C) RNA-LNP needed to be euthanized (Fig. 7a). As expected, no measurable HAI activity against the $\mathrm{H} 5 \mathrm{~N} 1$ virus was observed in mice immunized with the A/California/07/2009 HA mRNA-LNP vaccine (Fig. $7 \mathrm{~b}$ ). Interestingly, sera from these animals also did not display neutralization activity against the $\mathrm{H} 5 \mathrm{~N} 1$ virus in in vitro $\mathrm{MN}$ experiments (Fig. 7c).

\section{Discussion}

Currently approved influenza virus vaccines offer protection against well-matched circulating strains. These regimens mostly elicit antibody responses against the continuously changing immunodominant globular HA head domain. There is a need to develop "universal" influenza virus vaccines that induce potent immune responses against conserved viral epitopes and offer protection from heterologous and heterosubtypic strains. There are several viral protein regions that are conserved among different influenza virus strains, including epitopes in the HA stalk domain, neuraminidase, the ectodomain of the ion channel M2, matrix protein, and nucleoprotein (reviewed in refs. ${ }^{25,26}$ ). Vaccines against these targets might be able to elicit broad and protective influenza virus-specific immune responses (reviewed in ref. ${ }^{1}$ ). One of the most extensively studied targets is the immunosubdominant HA stalk (reviewed in ref. ${ }^{27}$ ). Seasonal influenza vaccines elicit poor antibody responses against $\mathrm{HA} \mathrm{stalk}^{28,29}$ in most individuals; thus, the development of new vaccine platforms 

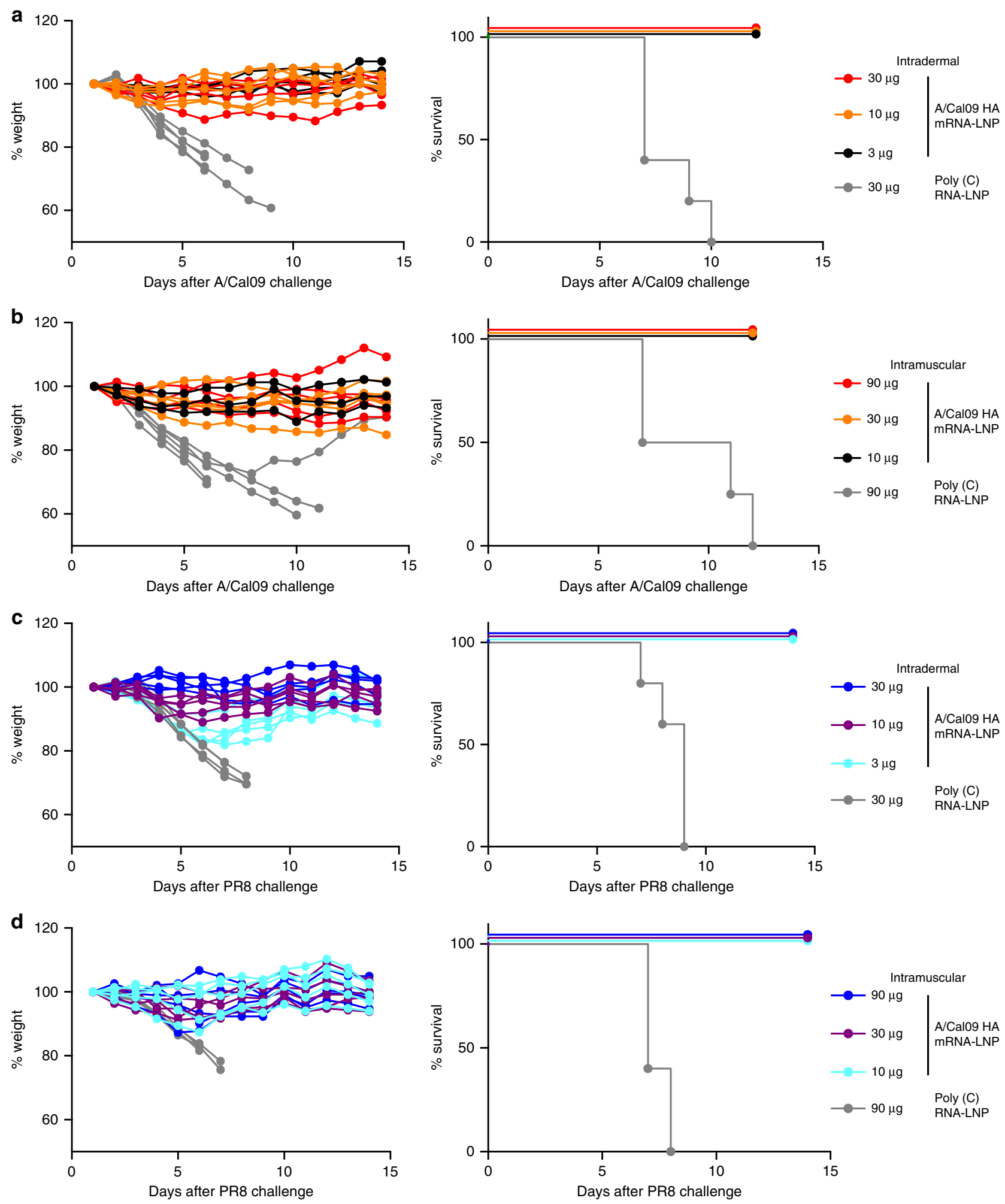

Fig. 5 Two immunizations with nucleoside-modified A/California/07/2009 HA mRNA-LNP vaccine elicits protection from A/California/07/2009 and Puerto Rico/8/1934 viruses. Mice received two i.d. (3, 10, or $30 \mu \mathrm{g})$ or i.m. (10, 30, or $90 \mu \mathrm{g}$ ) immunizations of A/California/07/2009 HA mRNA-LNPs or 30 (i.d.) or 90 (i.m.) $\mu$ g of poly(C) RNA-LNPs at week 0 (prime) and 4 (boost). Animals were challenged with lethal doses of homologous A/California/ 07/2009 (a, b) or heterologous A/Puerto Rico/8/1934 viruses (c, d) 5 weeks after the second immunization and weight loss and survival were followed. Two independent experiments were performed. $n=5$ mice and each weight loss line represents one animal 

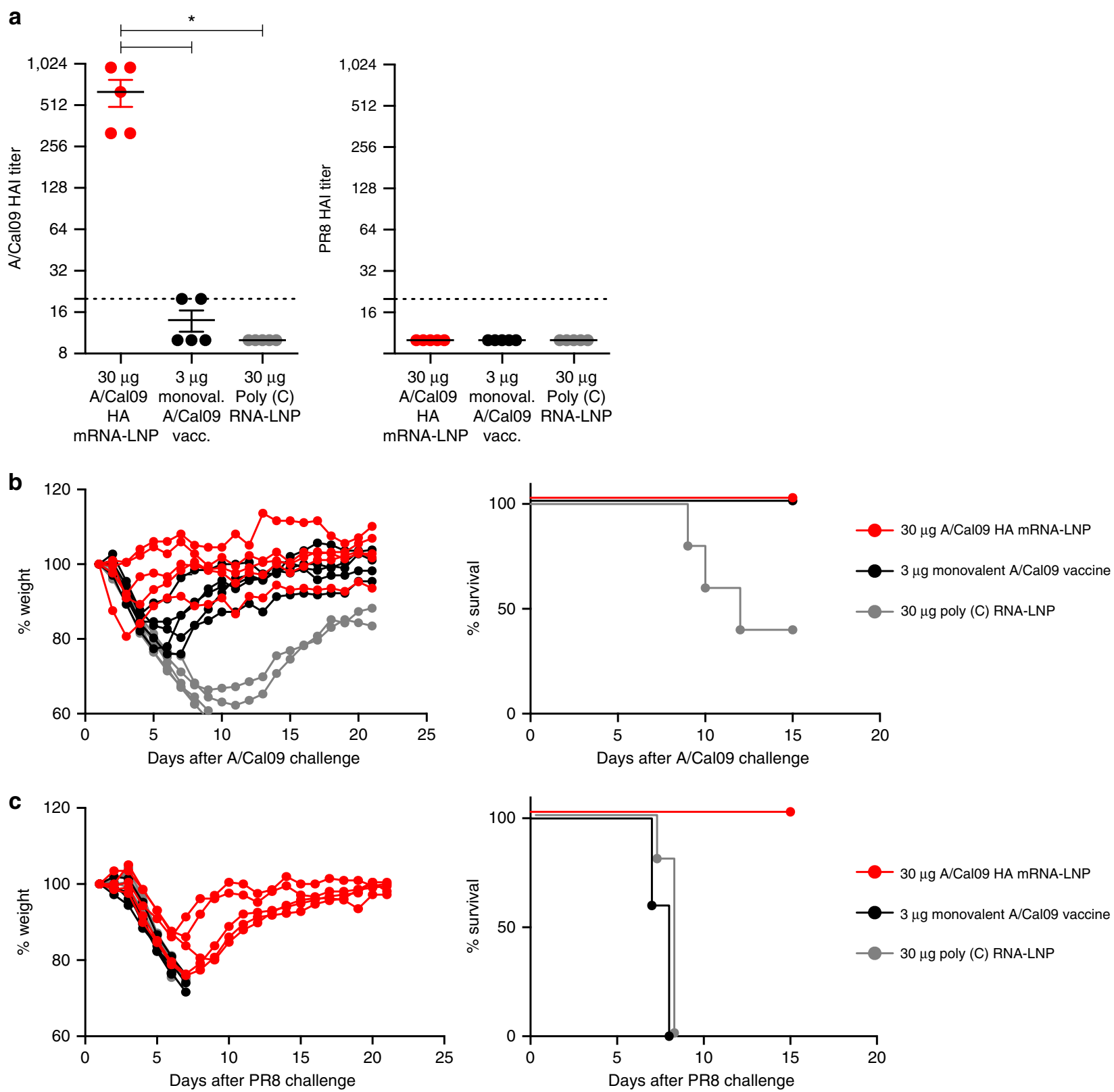

Fig. 6 Nucleoside-modified A/California/07/2009 HA mRNA-LNP vaccine elicits protection from A/California/07/2009 and A/Puerto Rico/8/1934 viruses after a single immunization. Mice received a single i.m. dose of $30 \mu \mathrm{g}$ A/California/07/2009 HA mRNA-LNPs. Control animals were vaccinated i. $\mathrm{m}$. with a single dose of $3 \mu \mathrm{g}$ of monovalent A/California/07/2009 virus vaccine or $30 \mu \mathrm{g}$ of poly(C) RNA-LNPs. HAI titers against the A/California/07/ 2009 and A/Puerto Rico/8/1934 virus (a) were determined 28 days post single immunization. Animals were challenged with lethal doses of homologous A/California/07/2009 (b) or heterologous A/Puerto Rico/8/1934 (c) viruses 28 days after immunization and weight loss and survival was followed. $n=5$ mice. a Horizontal lines show the mean; dotted line indicates the limit of detection. b, c Each weight loss line represents one animal. Statistical analysis: a one-way ANOVA with Bonferroni correction on log-transformed data, ${ }^{\star} p<0.05 ; \mathbf{b}$ two-way ANOVA with Bonferroni correction on weight loss graphs comparing A/California/07/2009 mRNA-immunized animals to inactivated virus-immunized animals. $p<0.05$ on days 4-6

and optimized immunogens that specifically elicit antibodies against this region is critically important.

Previous studies, using stable, headless HA proteins $\mathrm{s}^{3,4}$ or $\mathrm{cHA}$ encoding DNA and proteins (prime-boost) ${ }^{6}$ demonstrated that HA stalk-specific antibodies can be elicited in mice and ferrets. Yassine and colleagues developed headless H1 HA immunogens and demonstrated that three immunizations elicited protective antibodies against $\mathrm{H} 5 \mathrm{~N} 1$ influenza virus in mice and ferrets ${ }^{3}$. Impagliazzo and coworkers generated various monomeric, dimeric, and trimeric headless H1 mini HAs and showed that three immunizations with the best working trimeric construct resulted in protection against an $\mathrm{H} 5 \mathrm{~N} 1$ virus in mice ${ }^{4}$, and potent antibody responses in cynomolgus monkeys. Ermler and colleagues designed cHAs with HA heads from various influenza A viruses and HA stalks from influenza B viruses ${ }^{6}$. Sequential vaccination (one DNA prime and two protein boosts) with these constructs resulted in protection from influenza $B$ viruses in mice. A recent study from the same laboratory used sequential immunization in ferrets and demonstrated protection from pandemic $\mathrm{H} 1 \mathrm{~N} 1$ virus challenge ${ }^{7}$.

Here, we evaluated the efficacy of a newly developed influenza virus vaccine using nucleoside-modified, FPLC-purified fulllength HA-encoding mRNA encapsulated in $\mathrm{LNPs}^{30}$. A single immunization with $3 \mu \mathrm{g}$ of A/California/07/2009 HA mRNA- 

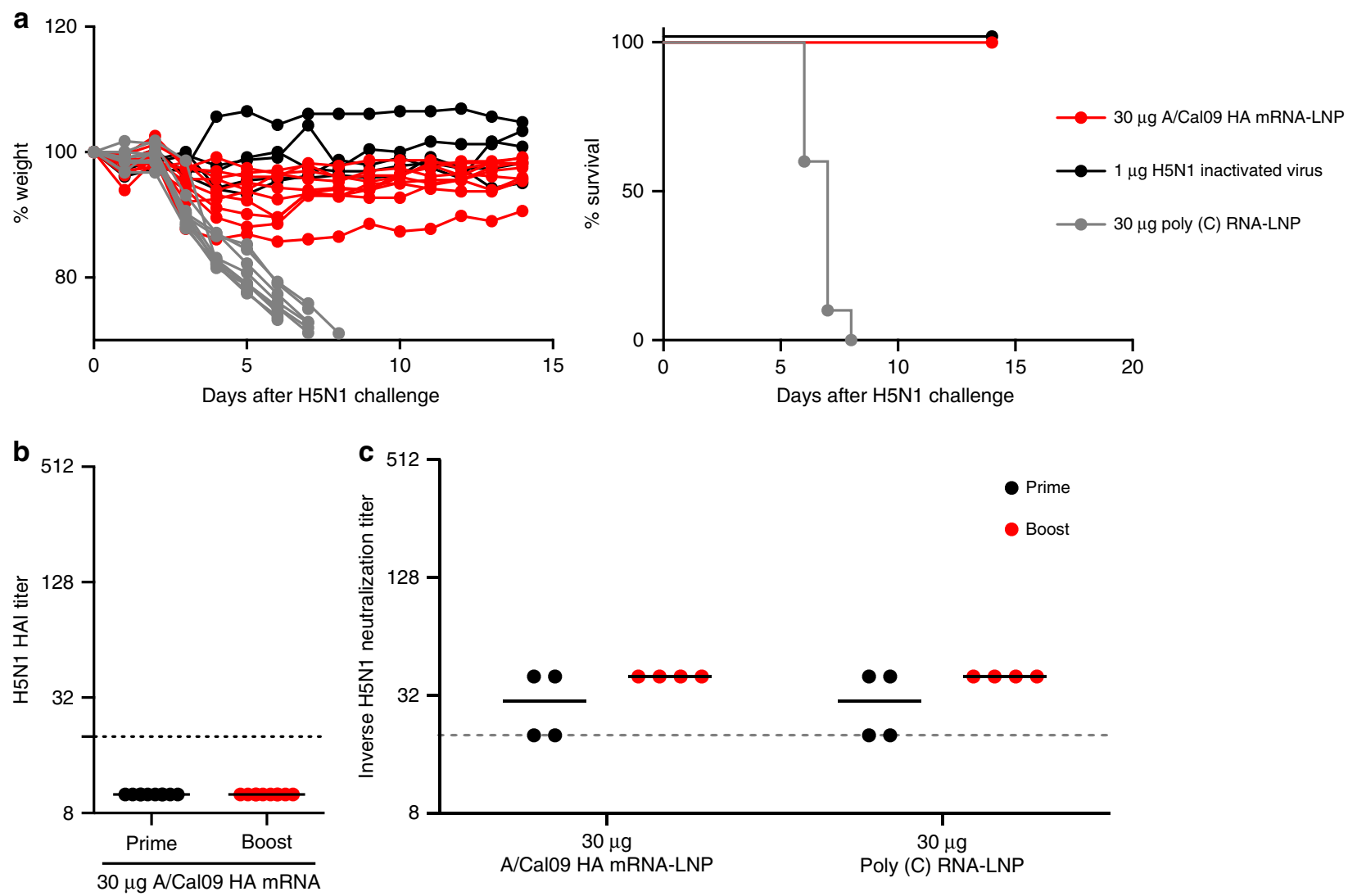

Fig. 7 Nucleoside-modified A/California/07/2009 HA mRNA-LNP vaccine elicits protection from the A/Vietnam/1203/04 (H5N1) virus after two immunizations. Mice received two i.d. doses of $30 \mu \mathrm{g}$ of A/California/07/2009 HA mRNA-LNPs at weeks 0 and 4. Control animals were vaccinated with two i.d. doses of $30 \mu \mathrm{g}$ of poly(C) RNA-LNPs or two i.m. doses of $1 \mu \mathrm{g}$ of inactivated H5N1 virus. (a) Animals were challenged with a lethal dose of A/Vietnam/1203/04 virus 28 days after the second immunization and weight loss and survival were followed. $n=10$ mice. HAI titers (b) and in vitro microneutralization activity (c) against the A/Vietnam/1203/04 virus were determined 28 days after the first and 28 days after the second immunization. Pooled serum samples were used for MN assays. a Each weight loss line represents one animal. b, c Horizontal lines show the mean; dotted line indicates the limit of detection. Statistical analysis: a two-way ANOVA with Bonferroni correction on weight loss graphs comparing A/California/07/2009 mRNAimmunized animals to inactivated virus-immunized animals, $p<0.05$ on days 5-7. $\mathbf{b}$ Unpaired $t$-test comparing post prime and post boost samples, ${ }^{\star} p<$ 0.05. c Two-way ANOVA with Bonferroni correction, ${ }^{*} p<0.05$

LNPs resulted in $\geq 1: 120 \mathrm{~A} /$ California/07/2009 $\mathrm{HAI}$ titers at 4 weeks post-immunization in mice. A second dose induced HAI values ranging between 1280-20,480 depending on the dose and the route of delivery (Fig. 1a, b). Of note, vaccination with A/ California/07/2009 mRNA-LNPs did not generate HAI activity against the A/Puerto Rico/8/1934 H1N1 influenza virus (Fig. 1c, d); however, the vaccine protected animals from A/Puerto Rico/8/ 1934 influenza virus challenge (Fig. 5c, d). This protection was likely mediated by HA stalk-reactive antibody responses that were measurable after a single immunization, and which were boosted by a subsequent immunization (Fig. 2).

A critical finding of this report is that a single immunization with A/California/07/2009 HA mRNA-LNPs resulted in protection against the homologous A/California/07/2009 and the heterologous A/Puerto Rico/8/1934 virus challenge in mice (Fig. 6b, c). Furthermore, we found that two immunizations with the A/California/07/2009 HA mRNA-LNP vaccine induced protection against $\mathrm{H} 5 \mathrm{~N} 1$ influenza virus infection (Fig. 7a). Of note, sera from these animals did not neutralize the challenge virus in in vitro $\mathrm{MN}$ experiments (Fig. 7c), which suggests a contribution of other effector immune mechanisms to heterosubtypic vaccine protection. These findings are in accordance with the literature as several recent studies demonstrated that the potency of HA stalkspecific antibodies were often enhanced by Fc receptor-mediated mechanisms such as antibody-dependent cell-mediated cytotoxicity or complement-dependent cytotoxicity ${ }^{31-34}$. Future studies will determine the correlates of protection after mRNALNP influenza vaccine administration. Several mRNA-based influenza vaccines have been described in recent years (reviewed in ref. ${ }^{35}$ ). For example, Bahl and colleagues demonstrated that a similar mRNA-LNP vaccine platform (using nucleoside-modified but not FPLC-purified H7 and H10 HA mRNAs) induced protection against the homologous influenza virus after a single dose immunization in mice and ferrets; however, no heterologous challenge data or evidence for the presence of stalk-specific antibodies was evaluated in these studies $^{18}$. Another recent report using unmodified HA mRNA-LNPs demonstrated durable induction of HA-specific antibodies in non-human primates, but again, the generation of crossprotective HA stalk antibodies was not reported ${ }^{36}$.

Ferrets are one of the best animal models for influenza virus research; however, a potential limitation of the use of ferrets for antiserum generation is that they usually generate more focused antibody responses to the variable head regions of $\mathrm{HA}$ than humans after natural infection ${ }^{37,38}$. We demonstrated that a single i.m. immunization with the A/California/07/2009 mRNALNP vaccine elicited $\geq 1: 40 \mathrm{HAI}$ activity against the A/California/ $07 / 2009$ virus in 8 out of 12 animals 4 weeks after vaccination (Fig. 4a). A subsequent immunization resulted in $\geq 1: 160 \mathrm{HAI}$ titers in 10 out of $12 \mathrm{~A} /$ California/07/2009 HA mRNA-LNP- 
immunized ferrets. The high variability in HAI activity may be due to the genetic heterogeneity of the out-bred animals, and thus it is possible that in some ferrets mRNA-LNP immunization skewed immune responses toward epitopes that neutralize viruses but do not induce HAI titers (Fig. 4a). The presence of stalkreactive antibodies (Fig. 4c) and MN titers against the homologous and heterologous H1N1 strains in A/California/07/2009 HA mRNA-LNP-immunized animals (Supplementary Fig. 5) support this hypothesis. Additionally, rabbits were immunized with the A/California/07/2009 HA mRNA-LNP vaccine and found that a single immunization elicited HA stalk-reactive antibodies (Supplementary Fig. 3), demonstrating that our findings in mice were translated to two species of large animals.

Here, we demonstrate that the nucleoside-modified, FPLCpurified influenza virus HA-encoding mRNA-LNP vaccine elicited HA stalk-specific antibody responses in mice, rabbits, and ferrets with durable HA stalk titers in mice. Future studies will address if mRNA-LNP immunization preferentially induce HA stalk-specific responses or they are elicited as a consequence of the very potent immune response without refocusing the immunodominance. Additional studies using adoptive serum transfer and $\mathrm{T}$ cell depletions will further evaluate the contribution of stalk-specific antibodies to vaccine protection in the heterosubtypic influenza virus infection model. The mRNA-LNP vaccine platform has additional beneficial features over other vaccines, including a favorable safety profile and highly scalable and potentially inexpensive manufacture. In addition to potency, from an influenza virus vaccine perspective, the most critical advantages of the present platform are the rapid development and the ease of production. It is notable that these vaccines are not subject to cell culture and egg-adaptive mutations that commonly arise as conventional influenza virus vaccines are manufactured 39,40 . The use of the nucleoside-modified mRNA-LNP platform with optimized HA stalk-inducing immunogens ${ }^{3,4,7}$ may offer a superior platform with easy clinical use. Currently available seasonal influenza virus vaccines do not protect well against antigenically drifted viral strains and they likely provide very little protection against emerging pandemic strains. Production of conventional, FDA-approved vaccines against new pandemic viruses could take at least 6 months, leaving the population unprotected during this period ${ }^{1}$. On the contrary, once the genetic sequence of the target HA antigen is known, mRNA-LNP vaccines can potentially be produced within weeks (reviewed in ref. ${ }^{12}$ ). mRNA-LNP vaccine production is sequence-independent and can be applied to virtually any pathogen. We believe that the data presented in this report combined with the additional beneficial features of nucleoside-modified and purified mRNA-LNPs makes the present platform a viable broadly protective influenza virus vaccine candidate.

\begin{abstract}
Methods
Ethics statement. The investigators faithfully adhered to the "Guide for the Care and Use of Laboratory Animals" by the Committee on Care of Laboratory Animal Resources Commission on Life Sciences, National Research Council. The animal facilities at the University of Pennsylvania, The Wistar Institute, the Icahn School of Medicine at Mount Sinai and Noble Life Sciences vivarium are fully accredited by the American Association for Accreditation of Laboratory Animal Care. All studies were conducted under protocols approved by the University of Pennsylvania, Noble Life Sciences, the Icahn School of Medicine at Mount Sinai, and The Wistar Institute IACUCs. The Wistar IACUC does not use weight loss as a criteria for euthanasia in murine influenza virus experiments.
\end{abstract}

mRNA production. mRNAs were produced as previously described ${ }^{41}$ using $\mathrm{T} 7 \mathrm{RNA}$ polymerase (Megascript, Ambion) on linearized plasmids (synthesized by GenScript) encoding codon-optimized ${ }^{42}$ Puerto Rico/8/1934 influenza virus HA (pTEV-PR8 HA-A101), A/California/7/2009 influenza virus hemmaglutinin (pTEV-A/Cal09 HAA101), and firefly luciferase (pTEV-Luc-A101). mRNAs were transcribed to contain
101 nucleotide-long poly(A) tails. One-methylpseudouridine (m1 $\left.{ }^{\Psi}\right)-5^{\prime}$-triphosphate (TriLink) instead of UTP was used to generate modified nucleoside-containing mRNA. RNAs were capped using the $\mathrm{m} 7 \mathrm{G}$ capping kit with $2^{\prime}$-O-methyltransferase (ScriptCap, Cellscript) to obtain capl. mRNA was purified by FPLC (Akta Purifier, GE Healthcare), as described ${ }^{43}$. All mRNAs were analyzed by denaturing or native agarose gel electrophoresis and were stored frozen at $-20^{\circ} \mathrm{C}$.

LNP formulation of the mRNA. FPLC-purified $\mathrm{m} 1 \Psi$-containing firefly luciferase and influenza virus HA-encoding mRNAs and poly(C) RNA (Sigma) were encapsulated in LNPs using a self-assembly process in which an aqueous solution of $\mathrm{mRNA}$ at $\mathrm{pH}=4.0$ is rapidly mixed with a solution of lipids dissolved in ethanol ${ }^{44}$. LNPs used in this study were similar in composition to those described previously ${ }^{44,45}$, which contain an ionizable cationic lipid (proprietary to Acuitas)/ phosphatidylcholine/cholesterol/PEG-lipid (50:10:38.5:1.5 mol/mol) and were encapsulated at an RNA to total lipid ratio of $\sim 0.05$ (wt/wt). They had a diameter of $\sim 80 \mathrm{~nm}$ as measured by dynamic light scattering using a Zetasizer Nano ZS (Malvern Instruments Ltd., Malvern, UK) instrument. mRNA-LNP formulations were stored at $-80^{\circ} \mathrm{C}$ at a concentration of mRNA of $\sim 1 \mu \mathrm{g} / \mu \mathrm{l}$.

Immunization of mice, rabbits, and ferrets. Mice: Female BALB/c mice aged 8 weeks were purchased from Charles River Laboratories. mRNA-LNPs were diluted in phosphate-buffered saline (PBS) and injected into animals intradermally with a $3 / 10 \mathrm{cc} 29 \frac{1}{2} \mathrm{G}$ insulin syringe (BD Biosciences). Four sites of injection ( $30 \mu \mathrm{l}$ each) over the lower back were used. For intramuscular injections, mRNA-LNPs were diluted in PBS and injected into animals using a 3/10cc 291/2G insulin syringe. Monovalent A/California/7/2009 virus vaccine ( $3 \mu \mathrm{g}$ in $50 \mu \mathrm{l}$ ) (BEI Resources, NR$20347)$ and inactivated $\mathrm{A} / \mathrm{Vietnam} / 1203 / 04$ virus vaccine $(1 \mu \mathrm{g}$ in $50 \mu \mathrm{l})$ (the virus was grown in eggs and inactivated with $0.3 \%$ formalin) were intramuscularly injected into the quadriceps muscle of animals using a 3/10cc $29 \frac{1}{2} \mathrm{G}$ insulin syringe. Inactivated $\mathrm{H} 5 \mathrm{~N} 1$ whole-virus vaccine preparation was generated by concentration of A/Vietnam/1203/04 virus (vaccine strain, 6:2 re-assortant with PR8, polybasic cleavage site of HA was removed) via ultracentrifugation followed by inactivation with $0.03 \%$ formalin. Protein content was measured using the Bradford method and reflects total protein rather than HA concentration.

Rabbits: Female New Zealand White rabbits aged 6 weeks were purchased from Charles River Laboratories. mRNA-LNPs were diluted in PBS and injected into animals intradermally with a $3 / 10 \mathrm{cc} 29 \mathrm{G}$ syringe (BD Biosciences). Six sites of injection ( $45 \mu \mathrm{l}$ each) over the lower back were used.

Ferrets: 7-10-week-old male and female ferrets were purchased from Triple F Farms Inc. mRNA-LNPs were diluted in PBS and injected intramuscularly into the upper thigh $(100 \mu \mathrm{l})$.

Blood collection. Mice: Blood was collected prior to each immunization by submandibular bleeding. Blood was centrifuged for $10 \mathrm{~min}$ at $2000 \times g$ in an Eppendorf microcentrifuge and the serum was stored at $-80^{\circ} \mathrm{C}$ and used for ELISA, MN, and HAI assays.

Rabbits: Blood was obtained from the lateral saphenous vein under anesthesia. Blood was centrifuged for $10 \mathrm{~min}$ at $3000 \times g$ and the serum was stored at $-80^{\circ} \mathrm{C}$ and used for ELISA and HAI assays.

Ferrets: Blood was obtained from the vena cava under anesthesia. Blood was centrifuged for $10 \mathrm{~min}$ at $3000 \times g$ and the serum was stored at $-80^{\circ} \mathrm{C}$ and used for ELISA, MN, and HAI assays.

Antibody reagents for flow cytometry. The following antibodies were used for flow cytometry: anti-CD4 PerCP/Cy5.5 (Clone GK1.5, BioLegend), anti-CD3 APC Cy7 (Clone 145-2C11, BD Biosciences), anti-TNF-a PE-Cy7 (Clone MP6-XT22, BD Biosciences), anti-IFN- $\gamma$ AF700 (Clone XMG1.2, BD Biosciences), anti-IL-2 APC (Clone JES6-5H4, BD Biosciences).

Mouse splenocyte stimulation/staining and flow cytometry. Single cell suspensions from mouse spleens were made in complete medium. Splenocytes were washed once in PBS and resuspended in complete medium at $2 \times 10^{7}$ cells $/ \mathrm{ml} .2 \times$ $10^{6}$ cells $(100 \mu \mathrm{l})$ per sample were stimulated for $6 \mathrm{~h}$ at $37^{\circ} \mathrm{C}$ using two overlapping influenza virus HA (A/California/07/2009) peptide pools (peptides are 14-mers or 15-mers, with 11 amino acid overlaps, provided by BEI resources, NR-19244) at $2 \mu \mathrm{g} / \mathrm{ml}$ per peptide. Golgi Plug (brefeldin A, BD Biosciences) and Golgi Stop (monensin, BD Biosciences) were diluted 1:100 and 1:143 in complete medium, respectively, and $20 \mu \mathrm{l}$ from both were added to each sample to inhibit the secretion of intracellular cytokines after $1 \mathrm{~h}$ of stimulation. A PMA $(10 \mathrm{ng} / \mathrm{ml})$-ionomycin $(250 \mathrm{ng} / \mathrm{ml})$ (Sigma) stimulated sample and unstimulated samples for each animal were included.

After stimulation, cells were washed in PBS and stained using the LIVE/DEAD Fixable Aqua Dead Cell Stain Kit and then surface stained for CD4 $(2 \mu \mathrm{g} / \mathrm{ml})$. Antibodies were incubated with cells for $30 \mathrm{~min}$ at RT. Following surface staining, cells were washed in FACS buffer and fixed using the Cytofix/Cytoperm kit (BD Biosciences), according to the manufacturer's instructions. Following fixation, the cells were washed in the permeabilization buffer and incubated with antibodies against CD3 $(1.6 \mu \mathrm{g} / \mathrm{ml})$, TNF- $\alpha(1.6 \mu \mathrm{g} / \mathrm{ml})$, IFN $-\gamma(4 \mu \mathrm{g} / \mathrm{ml})$, and IL-2 $(4 \mu \mathrm{g} / \mathrm{ml})$ for $1 \mathrm{~h}$ at RT. Following staining, the cells were washed with the permeabilization 
buffer, fixed (PBS containing 1\% paraformaldehyde) and stored at $4{ }^{\circ} \mathrm{C}$ until analysis. Splenocytes were analyzed on a modified LSR II flow cytometer (BD Biosciences).

Recombinant influenza virus cHAs. Recombinant HAs including $\mathrm{H} 1 \mathrm{HAs}$ of A/ California/04/09 and A/Puerto Rico/8/34 and chimeric cH6/1 $1_{\text {Calss }}$ (an H6 head domain from $\mathrm{A} / \mathrm{mallard} / \mathrm{Sweden} / 81 / 02$ on top of an $\mathrm{H} 1$ stalk domain from A/ California/04/09 with a stabilizing mutation in the stalk domain) and $\mathrm{cH} 5 / 1_{\text {PR8 }}$ (an H5 head domain from A/Vietnam/1203/04 on top of an H1 stalk domain from A/ Puerto Rico/8/34) constructs were expressed in the baculovirus expression system, as previously described ${ }^{46-48}$. They were then purified via a hexahistidine tag and $\mathrm{Ni}$-nitrilotriacetic acid (NTA) resin.

Influenza virus challenge studies. Mice were anesthetized with isoflurane and intranasally challenged with $200,000 \mathrm{TCID}_{50}$ of A/California/07/2009 influenza virus (6:2 virus with $6 \mathrm{~A} / \mathrm{Puerto} \mathrm{Rico} / 8 / 1934$ internal genes and $\mathrm{A} / \mathrm{California} / 07 /$ $2009 \mathrm{HA}$ and NA) or $5000 \mathrm{TCID}_{50}$ of A/Puerto Rico/8/1934 influenza virus in $50 \mu \mathrm{l}$ PBS. The A/California/7/2009 HA possessed a D225G mutation to facilitate viral replication in mice.

H5N1 challenges were performed with a 6:2 A/Vietnam/1203/04 reassortant virus (6 A/Puerto Rico/8/1934 internal genes and A/Vietnam/1203/04 HA and NA with the polybasic cleavage site removed from the HA). Animals were anesthetized using ketamine/xylazine $(0.15 \mathrm{mg}$ ketamine and $0.03 \mathrm{mg}$ xylazine $)$ delivered intraperitoneally in a volume of $100 \mu$. The mice were then inoculated with $50 \mu \mathrm{l}$ of a virus dilution (in PBS) containing 5 murine $50 \%$ lethal doses $\left(\mathrm{mLD}_{50}\right)$ of virus.

Enzyme-linked immunosorbent assays. ELISA plates were coated overnight at $4{ }^{\circ} \mathrm{C}$ with $3 \mu \mathrm{g} / \mathrm{ml}$ of recombinant protein. ELISA plates were blocked with a $3 \%$ (w/vol) bovine serum albumin (BSA) solution in PBS for $2 \mathrm{~h}$. Plates were then washed three times with PBS-Tween $20(0.1 \%)$ and serial dilutions of sera (diluted in $1 \%$ BSA in PBS) were added to the plates. After $2 \mathrm{~h}$ of incubation, plates were washed and alkaline phosphatase-conjugated or horseradish peroxidase (HRP)conjugated secondary antibodies were added. After a $1 \mathrm{~h}$ incubation, plates were washed and a p-nitrophenyl phosphate or a 3,3',5,5'-tetramethylbenzidine (TMB) substrate (Seracare, product number 50-00-03) was added. For HRP-based ELISAs, $\mathrm{HCl}$ was then added to stop the TMB reaction, and absorbance at $405 \mathrm{~nm}$ was measured using a plate reader. Results were obtained from technical duplicates.

HAl assays. A/Puerto Rico/8/1934 and A/California/07/2009 HAI assays: Mouse sera were heat-treated for $30 \mathrm{~min}$ at $55^{\circ} \mathrm{C}$. Ferret sera were treated with receptor destroying enzyme (RDE) from Vibrio cholerae (Denka Seiken, Chuo-ku, Tokyo, Japan) for $2 \mathrm{~h}$ at $37^{\circ} \mathrm{C}$ and then heat-treated for $30 \mathrm{~min}$ at $55^{\circ} \mathrm{C}$. Rabbit sera were RDE-treated for $2 \mathrm{~h}$ at $37^{\circ} \mathrm{C}$, heat-treated for $30 \mathrm{~min}$ at $55^{\circ} \mathrm{C}$, and then absorbed with turkey erythrocytes. Titrations were performed in 96 -well round bottom plates (BD Biosciences). First, $5 \mu$ of sera were added to $95 \mu$ of PBS (1:20 dilution), then two-fold serial dilutions were performed up to 1:2560 in a volume of $50 \mu \mathrm{l}$. Next, four agglutinating doses of virus were added to a total volume of $100 \mu \mathrm{l}$. Finally, $12.5 \mu \mathrm{l}$ of turkey erythrocytes (Lampire) ( $2 \%$ ( $\mathrm{vol} / \mathrm{vol})$ solution) was added to each well, and mixed thoroughly. Agglutination was read after incubating for $1 \mathrm{~h}$ at RT. HAI titers were expressed as the inverse of the highest dilution that inhibited four agglutinating doses of influenza virus.

A/swine/Jiangsu/40/2011 and A/Vietnam/1203/04 HAI assays: Mouse and ferret sera were treated with three volumes (based on original sera volume) of RDE for $18 \mathrm{~h}$ at $37^{\circ} \mathrm{C}$. Three volumes (based on original serum volume) of $2.5 \%$ sodium citrate solution was then added to the RDE-treated serum samples and were then incubated at $56^{\circ} \mathrm{C}$ for $1 \mathrm{~h}$. Three volumes of PBS (based on original serum volume) were added to each sample for a final dilution of 1:10. Titrations were performed in 96-well round bottom plates (BD Biosciences). First, $50 \mu \mathrm{l}$ of RDE-treated serum was added to the first well, then two-fold serial dilutions were performed up to 1:2048. Next, eight agglutinating doses of virus were added to a total volume of $50 \mu \mathrm{l}$. Virus and sera were then incubated at RT for $30 \mathrm{~min}$ with shaking. Following this incubation, $50 \mu \mathrm{l}$ of chicken erythrocytes (Charles River Laboratories) $(0.5 \%$ (vol/vol) solution) was added to each well, and mixed thoroughly. Agglutination was read after incubating for $1 \mathrm{~h}$ at $4{ }^{\circ} \mathrm{C}$. HAI titers were expressed as the inverse of the highest dilution that inhibited four agglutinating doses of influenza virus.

All samples were run in at least technical duplicates. The challenge virus strains (detailed in "Influenza virus challenge studies") were used for HAI assays.

MN assays. Mouse and ferret sera were treated with three volumes (based on original sera volume) of RDE for $18 \mathrm{~h}$ at $37^{\circ} \mathrm{C}$. Three volumes (based on original serum volume) of $2.5 \%$ sodium citrate solution was then added to the RDE-treated serum samples and were then incubated at $56^{\circ} \mathrm{C}$ for $1 \mathrm{~h}$. Three volumes of PBS (based on original serum volume) were added to each sample for a final dilution of 1:10.

Madin-Darby Canine Kidney (MDCK) cells (ATCC number PTA-6500) maintained in complete Dulbecco's Modified Eagle Medium with the addition of $10 \%$ fetal bovine serum, $1 \%$ Pen/Strep, $1 \%$ of a $1 \mathrm{M}$ HEPES stock solution were plated at a concentration of $1.5 \times 10^{4}$ cells per well in a 96-well plate and left to grow overnight at $37^{\circ} \mathrm{C}, 5 \% \mathrm{CO}_{2}$ until they reached $80-90 \%$ confluency.
RDE-treated sera was serially diluted two-fold in $1 \times$ minimal essential medium (MEM; $10 \% 10 \times$ MEM, 1\% $200 \mathrm{mM}$ L-glutamine, $1.6 \%$ of a $7.5 \%$ sodium bicarbonate stock solution ( $\mathrm{pH} 7.5), 1 \%$ of a $1 \mathrm{M}$ 4-(2-hydroxyethyl)-1piperazineethanesulfonic acid (HEPES) stock solution, $1 \%$ of penicillin/ streptomycin antibiotic cocktail (Pen/Strep, Gibco), $0.6 \%$ of a 35\% BSA stock solution) containing $1 \mu \mathrm{g} / \mathrm{ml} \mathrm{L}$-1-tosylamide-2-phenylethyl chloromethyl ketone (TPCK)-treated trypsin. For ferret sera, half of the volume of the serial dilutions was incubated with $100 \times \mathrm{TCID}_{50}$ of each virus (at a $1: 1$ volume ratio) for $1 \mathrm{~h}$ at RT with shaking. The virus-sera mixture was then applied to $80-90 \%$ confluent MDCK cells after they had been washed one time with sterile, $1 \times$ PBS. The cells were then incubated with the virus-sera mixture for $1 \mathrm{~h}$ at $33^{\circ} \mathrm{C}$. After the incubation, the virus-ferret sera mixture was removed, cells were washed with $1 \times \mathrm{PBS}$, and then covered with the remaining half of the serial sera dilutions (supplemented with a 1:1 addition of $1 \times \mathrm{MEM}$ with $1 \mu \mathrm{g} / \mathrm{ml}$ TPCK-treated trypsin).

For mouse sera, the entire amount of sera was incubated with $50 \times \mathrm{TCID}_{50}$ of $\mathrm{H} 5 \mathrm{~N} 1$ (at a 1:1 ratio) for $1 \mathrm{~h}$ at RT with shaking. The virus-sera mixture was then applied to $80-90 \%$ confluent MDCK cells after they had been washed one time with sterile $1 \times \mathrm{PBS}$. The cells were then incubated with the virus-sera mixture for 3 days at $33^{\circ} \mathrm{C}$. The difference in incubation time for the ferret sera vs. mouse sera on MDCK cells in these assays was due to the limited volumes of sera from mouse studies.

All samples were run in at least technical duplicates.

Multiple sequence alignment and phylogenetic tree. Sequences of the fulllength HA protein of A/California/04/2009 (pH1N1), A/Michigan/45/2015, A/New Caledonia/20/1999, A/South Carolina/1/1918, A/swine/Jiangsu/40/2011 (asH1N1), A/Brisbane/59/2007, A/swine/4/Mexico/2009, A/swine/Aichi/10/2015, A/swine/ Guangxi/QZ5/2014, and A/swine/Ohio/A02216472/2017 were downloaded from the Global Initiative for Sharing of all Influenza Data (GISAID) and aligned using the online server, Clustal Omega (https://www.ebi.ac.uk/Tools/msa/clustalo/). The phylogenetic tree produced from this alignment was downloaded, rooted to A/ South Carolina/1/1918, and visualized using FigTree.

Mapping conservation of pH1N1 and asH1N1. The structure used for visualizing the conserved residues between pH1N1 and asH1N1 is PDB ID 3LZG ${ }^{49}$. An alignment of $\mathrm{pH} 1 \mathrm{~N} 1$ and asH1N1 whole-HA protein sequences (downloaded from GISAID) was used to determine $100 \%$ conserved residues in Chimeria v1.12. These residues were then mapped onto a monomer of 3LZG in red. The alignment of pH1N1 and asH1N1 was also uploaded to the LALIGN server (https://embnet. vital-it.ch/software/LALIGN_form.html) to determine percent identity and similarity between the two HA proteins.

Statistical analyses. Statistical analyses were performed with Excel and Prism 5.0f software. Data were compared using one-way and two-way ANOVA corrected for multiple comparisons (Bonferroni method) and unpaired $t$-test. Survival analyses were perfomed using the log-rank (Mantel-Cox) test. The A/California/07/2009 crystal structure (PDB ID 3UBN) was visualized using PyMOL software.

Data availability. All data are available within the article and its Supplementary Information file or from the authors upon request.

Received: 21 December 2017 Accepted: 13 June 2018

Published online: 22 August 2018

\section{References}

1. Krammer, F. \& Palese, P. Advances in the development of influenza virus vaccines. Nat. Rev. Drug Discov. 14, 167-182 (2015).

2. Krammer, F. \& Palese, P. Influenza virus hemagglutinin stalk-based antibodies and vaccines. Curr. Opin. Virol. 3, 521-530 (2013).

3. Yassine, H. M. et al. Hemagglutinin-stem nanoparticles generate heterosubtypic influenza protection. Nat. Med. 21, 1065-1070 (2015).

4. Impagliazzo, A. et al. A stable trimeric influenza hemagglutinin stem as a broadly protective immunogen. Science 349, 1301-1306 (2015).

5. Valkenburg, S. A. et al. Stalking influenza by vaccination with pre-fusion headless HA mini-stem. Sci. Rep. 6, 22666 (2016).

6. Ermler, M. E. et al. Chimeric hemagglutinin constructs induce broad protection against influenza B virus challenge in the mouse model. J. Virol. 91, e00286-17 (2017)

7. Nachbagauer, R. et al. A universal influenza virus vaccine candidate confers protection against pandemic $\mathrm{H} 1 \mathrm{~N} 1$ infection in preclinical ferret studies. NPJ Vaccines 2, 1-13 (2017).

8. Wei, C. J. et al. Induction of broadly neutralizing H1N1 influenza antibodies by vaccination. Science 329, 1060-1064 (2010). 
9. Babu, T. M. et al. Live attenuated H7N7 influenza vaccine primes for a vigorous antibody response to inactivated H7N7 influenza vaccine. Vaccine 32, 6798-6804 (2014).

10. Talaat, K. R. et al. A live attenuated influenza $\mathrm{A}(\mathrm{H} 5 \mathrm{~N} 1)$ vaccine induces longterm immunity in the absence of a primary antibody response. J. Infect. Dis. 209, 1860-1869 (2014)

11. Halliley, J. L. et al. High-affinity H7 head and stalk domain-specific antibody responses to an inactivated influenza H7N7 vaccine after priming with live attenuated influenza vaccine. J. Infect. Dis. 212, 1270-1278 (2015).

12. Pardi, N., Hogan, M. J., Porter, F. W. \& Weissman, D. mRNA vaccines-a new era in vaccinology. Nat. Rev. Drug Discov. 17, 261-279 (2018).

13. Pardi, N. et al. Expression kinetics of nucleoside-modified mRNA delivered in lipid nanoparticles to mice by various routes. J. Control Release 217, 345-351 (2015).

14. Pardi, N. et al. Zika virus protection by a single low-dose nucleoside-modified mRNA vaccination. Nature 543, 248-251 (2017).

15. Brazzoli, M. et al. Induction of broad-based immunity and protective efficacy by self-amplifying mRNA vaccines encoding influenza virus hemagglutinin. J. Virol. 90, 332-344 (2015).

16. Magini, D. et al. Self-amplifying mRNA vaccines expressing multiple conserved influenza antigens confer protection against homologous and heterosubtypic viral challenge. PLoS ONE 11, e0161193 (2016).

17. Chahal, J. S. et al. Dendrimer-RNA nanoparticles generate protective immunity against lethal Ebola, H1N1 influenza, and Toxoplasma gondii challenges with a single dose. Proc. Natl Acad. Sci. USA 113, E4133-E4142 (2016).

18. Bahl, K. et al. Preclinical and clinical demonstration of immunogenicity by mRNA vaccines against $\mathrm{H} 10 \mathrm{~N} 8$ and $\mathrm{H} 7 \mathrm{~N} 9$ influenza viruses. Mol. Ther. 25, 1316-1327 (2017).

19. Petsch, B. et al. Protective efficacy of in vitro synthesized, specific mRNA vaccines against influenza A virus infection. Nat. Biotechnol. 30, 1210-1216 (2012).

20. Kariko, K. et al. Incorporation of pseudouridine into mRNA yields superior nonimmunogenic vector with increased translational capacity and biological stability. Mol. Ther. 16, 1833-1840 (2008).

21. Kariko, K., Muramatsu, H., Ludwig, J. \& Weissman, D. Generating the optimal mRNA for therapy: HPLC purification eliminates immune activation and improves translation of nucleoside-modified, protein-encoding mRNA. Nucleic Acids Res. 39, e142 (2011).

22. Pica, N. et al. Hemagglutinin stalk antibodies elicited by the 2009 pandemic influenza virus as a mechanism for the extinction of seasonal H1N1 viruses. Proc. Natl Acad. Sci. USA 109, 2573-2578 (2012).

23. Swain, S. L. et al. CD4+ T-cell memory: generation and multi-faceted roles for $\mathrm{CD} 4+\mathrm{T}$ cells in protective immunity to influenza. Immunol. Rev. 211, 8-22 (2006).

24. Krammer, F., Pica, N., Hai, R., Margine, I. \& Palese, P. Chimeric hemagglutinin influenza virus vaccine constructs elicit broadly protective stalk-specific antibodies. J. Virol. 87, 6542-6550 (2013).

25. Krammer, F., Garcia-Sastre, A. \& Palese, P. Is it possible to develop a "universal" influenza virus vaccine? Toward a universal influenza virus vaccine: potential target antigens and critical aspects for vaccine development. Cold Spring Harb. Perspect. Biol. 10, a028845 (2017).

26. Andrews, S. F., Graham, B. S., Mascola, J. R. \& McDermott, A. B. Is it possible to develop a "universal" influenza virus vaccine? Immunogenetic considerations underlying B-cell biology in the development of a pan-subtype influenza A vaccine targeting the hemagglutinin stem. Cold Spring Harb. Perspect. Biol. 10, a029413 (2017).

27. Krammer, F. Novel universal influenza virus vaccine approaches. Curr. Opin. Virol. 17, 95-103 (2016).

28. Corti, D. et al. Heterosubtypic neutralizing antibodies are produced by individuals immunized with a seasonal influenza vaccine. J. Clin. Invest. 120, 1663-1673 (2010).

29. Sui, J. et al. Wide prevalence of heterosubtypic broadly neutralizing human anti-influenza A antibodies. Clin. Infect. Dis. 52, 1003-1009 (2011).

30. Pardi, N. \& Weissman, D. Nucleoside modified mRNA vaccines for infectious diseases. Methods Mol. Biol. 1499, 109-121 (2017).

31. DiLillo, D. J., Tan, G. S., Palese, P. \& Ravetch, J. V. Broadly neutralizing hemagglutinin stalk-specific antibodies require FcgammaR interactions for protection against influenza virus in vivo. Nat. Med. 20, 143-151 (2014).

32. Henry Dunand, C. J. et al. Both neutralizing and non-neutralizing human H7N9 influenza vaccine-induced monoclonal antibodies confer protection. Cell Host Microbe 19, 800-813 (2016).

33. Leon, P. E. et al. Optimal activation of Fc-mediated effector functions by influenza virus hemagglutinin antibodies requires two points of contact. Proc. Natl Acad. Sci. USA 113, E5944-E5951 (2016).

34. Terajima, M. et al. Complement-dependent lysis of influenza a virus-infected cells by broadly cross-reactive human monoclonal antibodies. J. Virol. 85, 13463-13467 (2011).
35. Scorza, F. B. \& Pardi, N. New kids on the block: RNA-based influenza virus vaccines. Vaccines 6, E20 (2018)

36. Lutz, J. et al. Unmodified mRNA in LNPs constitutes a competitive technology for prophylactic vaccines. NPJ Vaccines 2, 1-9 (2017).

37. Li, Y. et al. Immune history shapes specificity of pandemic H1N1 influenza antibody responses. J. Exp. Med. 210, 1493-1500 (2013).

38. Nachbagauer, R. et al. Defining the antibody cross-reactome directed against the influenza virus surface glycoproteins. Nat. Immunol. 18, 464-473 (2017).

39. Zost, S. J. et al. Contemporary $\mathrm{H} 3 \mathrm{~N} 2$ influenza viruses have a glycosylation site that alters binding of antibodies elicited by egg-adapted vaccine strains. Proc. Natl Acad. Sci. USA 114, 12578-12583 (2017).

40. Wu, N. C. et al. A structural explanation for the low effectiveness of the seasonal influenza H3N2 vaccine. PLoS Pathog. 13, e1006682 (2017).

41. Pardi, N., Muramatsu, H., Weissman, D. \& Kariko, K. In vitro transcription of long RNA containing modified nucleosides. Methods Mol. Biol. 969, 29-42 (2013).

42. Thess, A. et al. Sequence-engineered mRNA without chemical nucleoside modifications enables an effective protein therapy in large animals. Mol. Ther. 23, 1456-1464 (2015).

43. Weissman, D., Pardi, N., Muramatsu, H. \& Kariko, K. HPLC purification of in vitro transcribed long RNA. Methods Mol. Biol. 969, 43-54 (2013).

44. Maier, M. A. et al. Biodegradable lipids enabling rapidly eliminated lipid nanoparticles for systemic delivery of RNAi therapeutics. Mol. Ther. 21, 1570-1578 (2013)

45. Jayaraman, M. et al. Maximizing the potency of siRNA lipid nanoparticles for hepatic gene silencing in vivo. Angew. Chem. Int. Ed. Engl. 51, 8529-8533 (2012).

46. Krammer, F. et al. A carboxy-terminal trimerization domain stabilizes conformational epitopes on the stalk domain of soluble recombinant hemagglutinin substrates. PLoS ONE 7, e43603 (2012).

47. Hai, R. et al. Influenza viruses expressing chimeric hemagglutinins: globular head and stalk domains derived from different subtypes. J. Virol. 86, 5774-5781 (2012).

48. Margine, I., Palese, P. \& Krammer, F. Expression of functional recombinant hemagglutinin and neuraminidase proteins from the novel H7N9 influenza virus using the baculovirus expression system. J. Vis. Exp. 81, e51112 (2013).

49. Xu, R. et al. Structural basis of preexisting immunity to the $2009 \mathrm{H} 1 \mathrm{~N} 1$ pandemic influenza virus. Science 328, 357-360 (2010).

\section{Acknowledgements}

We thank Michael J. Hogan, Ph.D., and Istvan Tombacz, Ph.D., for assistance with statistical analysis and critical review of the manuscript. S.E.H. was supported by the National Institute of Allergy and Infectious Diseases (1R01AI113047, 1R01AI108686, and Centers of Influenza Virus Research and Surveillance (CEIRS) contract HHSN272201400005C). S.E.H. holds an Investigators in the Pathogenesis of Infectious Disease Award from the Burroughs Wellcome Fund. D.W. was supported by the NIAID of the NIH under award numbers R01-AI050484, R01-AI124429, and R01-AI084860, and the New Frontier Sciences division of Takeda Pharmaceuticals. Work in the Krammer Laboratory was supported by by NIAID U19 AI109946 and the Centers of Influenza Virus Research and Surveillance (CEIRS) contract HHSN272201400008C.

\section{Author contributions}

N.P., D.W., K.P., S.E.H., and F.K. designed vaccine studies. N.P., K.P., M.M., and E.L.K performed the studies. S.J.Z. generated Fig. S1. B.L.M., Y.K.T., T.D.M., F.K., C.J.B., and M.J.H. supplied reagents. N.P., D.W., S.E.H., F.K., and K.K. wrote the manuscript with help from the co-authors.

\section{Additional information}

Supplementary Information accompanies this paper at https://doi.org/10.1038/s41467 018-05482-0.

Competing interests: In accordance with the University of Pennsylvania policies and procedures and our ethical obligations as researchers, we report that K.K. and D.W. are named on patents that describe the use of nucleoside-modified mRNA as a platform to deliver therapeutic proteins. D.W. and N.P. are also named on a patent describing the use of modified mRNA in lipid nanoparticles as a vaccine platform. We have disclosed those interests fully to the University of Pennsylvania, and we have in place an approved plan for managing any potential conflicts arising from licensing of our patents. S.E.H. has received consultancy fee from Lumen, Novavax, and Merck for work unrelated to this report. The remaining authors declare no competing interests.

Reprints and permission information is available online at http://npg.nature.com/ reprintsandpermissions/

Publisher's note: Springer Nature remains neutral with regard to jurisdictional claims in published maps and institutional affiliations. 
(cc) (i) Open Access This article is licensed under a Creative Commons Cy Attribution 4.0 International License, which permits use, sharing, adaptation, distribution and reproduction in any medium or format, as long as you give appropriate credit to the original author(s) and the source, provide a link to the Creative Commons license, and indicate if changes were made. The images or other third party material in this article are included in the article's Creative Commons license, unless indicated otherwise in a credit line to the material. If material is not included in the article's Creative Commons license and your intended use is not permitted by statutory regulation or exceeds the permitted use, you will need to obtain permission directly from the copyright holder. To view a copy of this license, visit http://creativecommons.org/ licenses/by/4.0/.

(C) The Author(s) 2018 